\title{
Understanding the Learning Process in Down Syndrome by Analyzing the Gene Ontology Result of Two Different Drugs
}

Handan Kulan ( $\sim$ handankulan@beykoz.edu.tr)

Beykoz University

\section{Research Article}

Keywords:

Posted Date: January 17th, 2022

DOI: https://doi.org/10.21203/rs.3.rs-1229052/v1

License: (c) (1) This work is licensed under a Creative Commons Attribution 4.0 International License.

Read Full License 


\title{
Understanding the Learning Process in Down Syndrome by Analyzing the Gene Ontology Result of Two Different Drugs
}

\author{
Handan Kulan"
}

\section{ABSTRACT}

Down syndrome (DS) is considered as the most prevalent cause of mental retardation. Possible causes of DS are nondisjunction of human chromosome 21 (Hsa21) or a Robertsonian translocation between Hsa21 and another chromosome. Scientists are trying to develop drugs to rescue learning performance in DS disease. In the last few years, a large number of drugs and small molecules have been shown to recover one or more abnormalities in transgenic mouse models. In this study, the effect of memantine and $\mathbf{R O 4 9 3 8 5 8 1}$ drugs on rescued learning was determined by evaluating the protein expression level of the transgenic mouse model using machine learning algorithm techniques. Gene set enrichment analysis (GSEA) was performed and both drugs were found to have an effect on common gene ontology (GO) terms. Thanks to the applied methodology and the result of this study, it may be possible to diagnose and treat the disease by developing a new drug that stimulates areas where the common GO terms are precisely determined.

\section{Introduction}

DS is the genetic disorders that lead to intellectual disability (ID). British doctor John Langdon Down reported its clinical description in 1866 and in 1959 Jerome Lejeune emphasized the association of DS with an extra copy of HSA $21^{1}$. According to recent genomic sequence annotation ${ }^{2}$, HSA2 1 encodes 550 genes. Of these, the third copy of HSA21 is accountable for almost 160 protein-coding genes and five microRNAs. Even if Down syndrome features have largely been attributed to the overexpression of HSA21 genes ${ }^{3}$, noncoding element ${ }^{4}$ or DNA methylation ${ }^{5}$ play role in DS phenotypes. The characteristics features of DS people are defects in learning, language and motor skills, mental retardation, short stature, heart defects, morphological abnormalities of the head and limbs. DS people are at risk of developing certain types of blood diseases, such as leukemia, autoimmune disorders and Alzheimer's disease (AD). Due to its high significance, DS, various aspects of the disease have been investigated by genomic studies. However, dysregulations in the key molecular networks in DS are still unknown. The critical problem facing the researchers is the correlation of the increased level of expression of specific Hsa21 genes with the development of defects in learning or memory $(\mathrm{L} / \mathrm{M})^{6-10}$.

Remarkable researches has been conducted on mouse models of improving cognitive function in DS for therapeutic drugs. Scientists are trying to find DS factors and possible treatments by evaluating the protein levels of mouse models. However, it is cumbersome to model DS in mice as the orthologs of Hsa21 genes map to three mouse chromosomes (Mmu10, Mmu16, and Mmu17) ${ }^{11}$. So far, the mouse models created are partially trisomic, which means that most of them contain an extra copy, but not all mouse genes are equivalent to Hsa21. Using mouse models, protein expression profiles of DS patients are analyzed to assess potential perturbations in pathways known or predicted to be associated with $\mathrm{L} / \mathrm{M}$ abnormalities seen in DS patients ${ }^{12}$. However, with the studies conducted so far, effective pharmacotherapies for treating learning disabilities in DS have not been found ${ }^{13}$. Scientists are trying to develop new drugs by studying the interactions between proteins themselves and the possible interactions they establish with drugs. Comparison of protein profiles between the transgenic mice when learning is unsuccessful and rescued with drugs helps to decipher the learning process in DS. Untreated transgenic mice are not able to learn, but they can successfully learn if the drug is injected, and thus learning is rescued. These achievements have increased interest in clinical trials to develop new drugs ${ }^{14-17}$. Memantine drug is currently used to treat learning disabilities in DS. Memantine modulates excitatory neurotransmission through antagonizing activity of Nmethyl-D-aspartate (NMDA) receptor. It has also been suggested that increased GABAA -mediated inhibition is a crucial mechanism for the $\mathrm{L} / \mathrm{M}$ changes found in transgenic mice ${ }^{18-20}$. Drug 3-bromo-10(difluoromethyl)-9H-benzo[f]imidazo[1,5-a][1,2,4]triazolo[1,5-d][1,4]diazepine (RO4938581) is GABAA receptor negative allosteric modulator (NAM) $)^{21,22}$ and like memantine, is used to rescue protein anomalies in mouse models.

* Beykoz University, Software Engineering, Beykoz, TR. 
The performance of rescued drugs in DS has been studied by different scientists. Ahmed et $\mathrm{al}^{23}$ performed a three level mixed effects (3LME) statistical analysis model of the trisomic and normal mouse protein profiles exposed to and not exposed to memantine. They showed that more than half of the protein levels changed significantly in the hippocampus when exposed to contextual fear conditioning (CFC) or memantine. They also noted significant differences between the profiles of normal and Ts65Dn mice, which are $55 \%$ orthologous for a region that shows a perfectly conserved linkage with Hsa21 and shows many DS-related features. However, the standard statistical analyzes applied in the studies do not identify many important features, for example, whether the changes caused by the drug are critical for improving learning performance. Machine learning methods correct the shortcomings encountered in standard statistical methods. Thus, Higuera et $\mathrm{al}^{24}$ analyzed the protein profiles using unsupervised learning, Self Organizing Map (SOM) to find the proteins expressed differently by the memantine. They used the Wilcoxon rank-sum test to identify proteins whose levels are significantly different between classes of mice. They analyzed the expression levels of 77 proteins obtained from both memantine-treated and untreated normal genotype control mice and their trisomic littermates (Ts65Dn). With the selected proteins, mice in failed learning were efficiently distinguished from mice in rescued learning. Also, Block et $\mathrm{al}^{22}$ used GABAA $\alpha 5$ selective modulator, RO4938581, for rescuing protein anomalies in the Ts65Dn. In their work, 91 protein levels relevant to brain functions were measured by applying $3 \mathrm{LME}$ and of the 52 anomalies in Ts65Dn, 44 have been corrected by RO493858. Showing the critical factors in discriminating mice in rescued and failed learning, these selected subsets of proteins were compared to understand the effects of drugs on protein expression. This type of analysis can help address questions such as whether Ts65Dn mice treated with different drugs show similar responses. Rather than statistical techniques used in Block et al research, Kulan et $\mathrm{al}^{25}$ applied the forward feature selection method to select subsets from two datasets showing variation in protein expression level interacting with memantine or RO4938581. They emphasized that two drugs, memantine and RO4938581, affect the common proteins.

Until now, critical proteins related to L/M abnormalities seen in DS have been found by applying statistical techniques or machine learning algorithms. In this study, in addition to those conducted in previous studies, after significant subsets of proteins were selected from the memantine and RO4938581 datasets, gene ontology analysis was also performed to find common GO terms in which both drugs act. The association of GO terms with the proteins provides an understanding of the biological processes to which proteins contribute. Identification of common gene terms will help to identify biological processes that play a key role in the recovery of learning performance in DS. Analyzing these processes contributes to our understanding of the learning process and the design of new drugs for treatment.

A

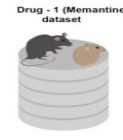

Control and trisomic
(Down syndrame) mice
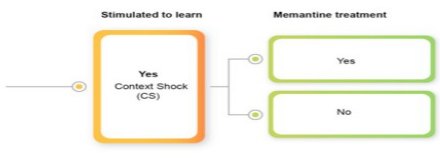

(2)

$\mathbf{B}$
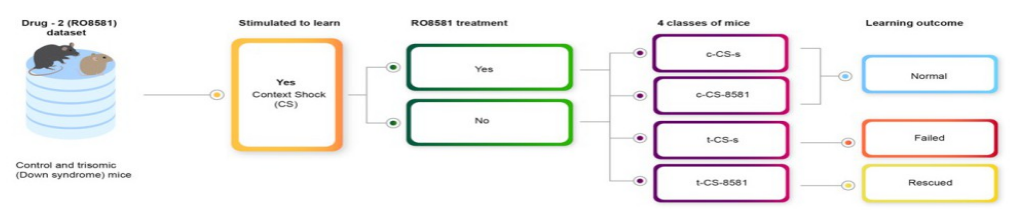

C

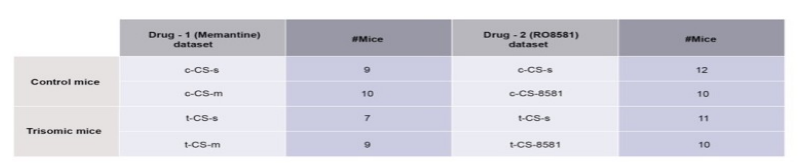

D

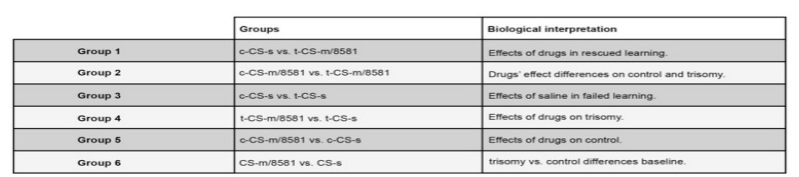

Fig 1. | a, Memantine dataset (Context-Shock, CS, control, c, trisomic, t, saline, s and memantine, $\mathrm{m}$ ).

b, R0 dataset (8581, RO4938581). c, Class and number of mice. d, Name and biological interpretation of groups. 


\section{Results}

\section{Framework overview.}

The RO4938581 and memantine drug datasets consisting of the protein expression profiles of Ts65Dn mice and their control littermates were examined and contents are shown in Fig. 1. The memantine dataset (Fig. 1a) consists of the expression level of 77 proteins at which signals were detected in the nuclear fraction of the cortex. Protein lysates were prepared from the brains of three month old male Ts65Dn model mice and their controls. 15 samples (three replicates of a five-point dilution series) were taken from each mouse. The dataset is divided into four classes of mice, depending on their genotype, CS exposure and applied drugs. In CFC protocols ${ }^{26}$, CS-class mice are placed in a cage and waited for a few minutes to explore the context. Wild-type mice are expected to associate context with an electric shock and freeze after repeated exposure to the same cage. However, the Ts65Dn CS class mice cannot learn and freeze. Learning can be rescued in this class of mice if memantine is injected. The dataset of RO493858 (Fig. 1b) shows the level of protein expression measured from the whole lysate hippocampus of Spanish Ts65Dn ten-month-old mice treated with RO4938581 or saline as a control vehicle. Each sample is associated with three replicates of a five point dilution series. The dataset was divided into four classes, covering control and Ts65Dn mice treated with saline or RO4938581. The expression levels of proteins were measured with reverse phase protein arrays $(\mathrm{RPPA})^{27}$ which provides a quantitative analysis of the differential expression of proteins. For in-depth analysis of datasets, the datasets were divided into six groups (Fig. 1d) and was used to find the common GO terms that both drugs affect. To this end, a framework (Fig. 2) developed using a data-driven, supervised learning modeling approach to understand drug effects on DS. Fig. 2 shows all the methods applied in this research in stages to evaluate the GO terms affected by the drug memantine and RO4938581.

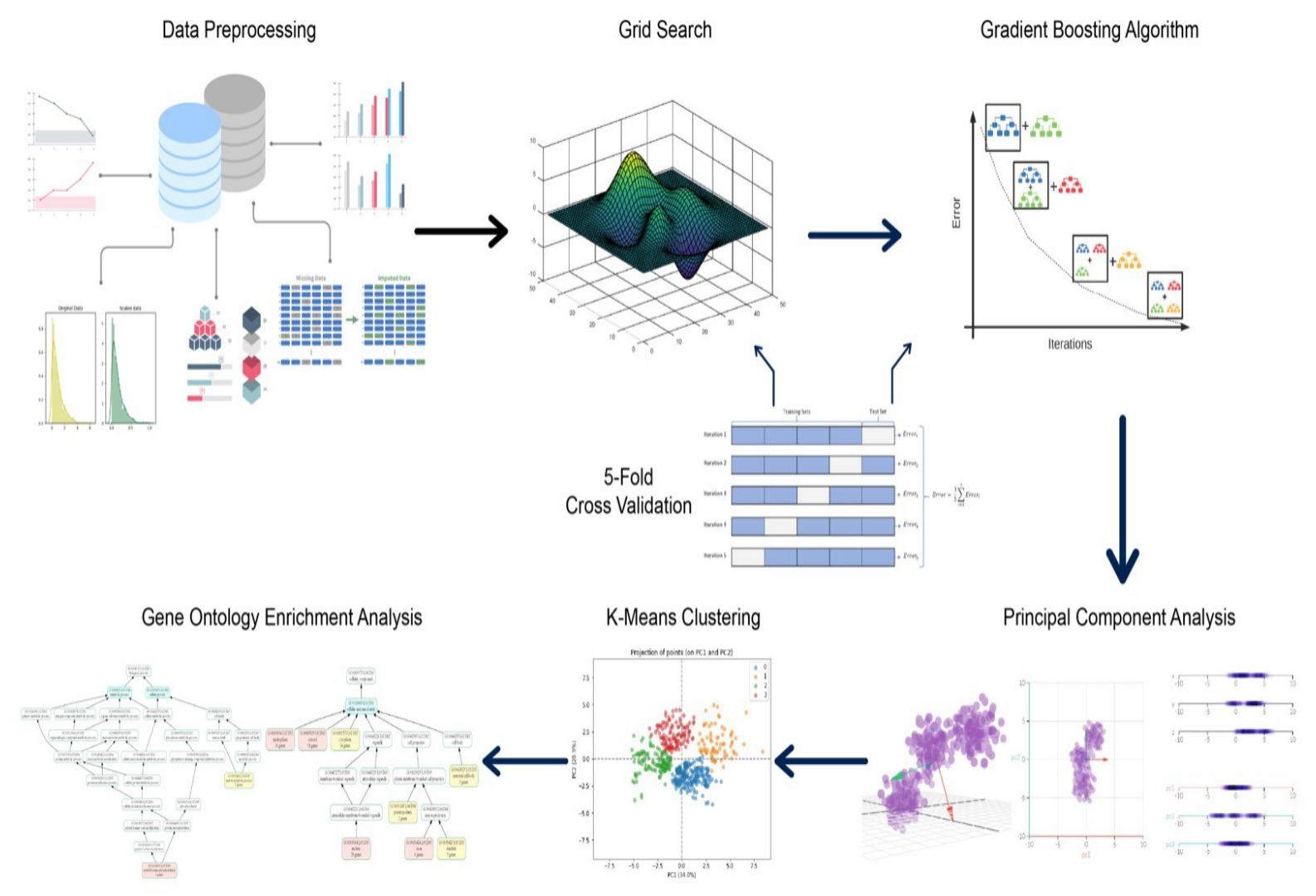

Fig 2. An illustration of the proposed framework. The methods used, respectively, are : Data preprocessing step, grid search method for selecting parameter combination, gradient boosting classifier algorithm for selecting the significant protein subset for each group, PCA analysis for projection data onto 2D axes, Kmeans cluster algorithm for clustering data and GSEA for finding common GO terms. 
Firstly, at the preprocessing step, the missing values were filled in and the datasets were normalized. After preprocessing step, four components of the framework were generated by an algorithmic approach. A gradient boosting algorithm was applied to select protein subsets affected by RO4938581 or memantine drugs. Using the grid search method in the gradient boosting algorithm, eight different models were created for different combination of hyperparameter values and a combination of parameters was determined for each model, which gave the best accuracy result. Eight different models were run with the gradient boosting classification algorithm and the best model was selected for each group (see Fig. 1d) according to the PCA cluster analysis result. In PCA, the selected proteins were projected onto reduced 2D axes. The Kmeans algorithm was applied for clustering proteins and then the obtained score was analyzed. Based on the highest accuracy score, the most accurate model was selected from eight models for each group. After determining the most important subset of proteins for each group, the common gene terms by which both drugs act on the proteins contained in the two datasets were studied by performing GSEA.

In the treatment of diseases, the causes of the disease can be determined in GO terms that describe the molecular functions, processes and subcellular localization of gene drug targets. Diseases can be treated by defining GO terms, and DS defects can be treated by developing a drug that acts on areas specified in GO terms. The ultimate goal is to understand the potential target to develop therapeutics for DS. But, it is a difficult task to identify and interpret the causes and consequences of trisomy-induced changes in HSA21 and non-HSA21 gene expression, because the number and identity of genes with altered expression differs between experiments. However, in this study, after selecting the most important gene products using machine learning algorithms, effective GO terms were determined by taking into account the protein-coding genes at the latest stage. It is thought that the effective GO terms obtained in this study will make an important contribution to the diagnosis and treatment of DS.

\section{Scenario analysis.}

For each group shown in Fig. 1d, eight different models with different parameter combinations were determined using grid search method. The combination of parameters was determined based on the smallest average absolute error obtained for the test set result of the grid search. Then, parameter combinations of eight models for each group were run with gradient boosting algorithm and eight different protein subsets were obtained for each group. The most important subset of proteins was determined according to the error rate result of the PCA cluster algorithm. Table 1 and Table 2 show the parameter combination of selected protein subset for each group in memantine dataset and the RO4938581 dataset, respectively.

Table 1. The best combination of parameter values for each group in the memantine dataset

\begin{tabular}{c|c|c|c|c|c|c|}
\hline & Group 1 & Group 2 & Group 3 & Group 4 & Group 5 & Group 6 \\
\cline { 2 - 7 } n_estimators & 350 & 600 & 520 & 400 & 330 & 750 \\
\hline max_depth & 3 & 6 & 15 & 3 & 5 & 3 \\
\hline min_samples_leaf & 3 & 1 & 1 & 3 & 0.05 & 0.05 \\
\hline learning_rate & 0.05 & 0.04 & 0.05 & 3 & 10 & 2 \\
\hline min_samples_split & 2 & 7 & 5 & Is & Is & huber \\
\hline Ioss & Is & Is & Is & 0.5 & 0.3 & 0.3 \\
\hline subsample & 0.3 & 0.3 & 0.3 & & & \\
\hline
\end{tabular}

The hyperparameters of the gradient boosting modeling are the number of trees to be modeled (n_estimators), the maximum depth of each tree (max_depth), the minimum number of samples required to be at a leaf node (min_samples_leaf), the learning rate (learning_rate), the minimum number of samples required to split an internal node (min_samples_split), the loss function to be optimized (loss), the fraction of samples to be used for fitting the individual base learners (subsample).

Table 2. The best combination of parameter values for each group in the RO4938581 dataset

\begin{tabular}{|c|c|c|c|c|c|c|}
\hline & Group 1 & Group 2 & Group 3 & Group 4 & Group 5 & Group 6 \\
\cline { 2 - 7 } n_estimators & 350 & 800 & 450 & 730 & 800 & 900 \\
\hline max_depth & 3 & 15 & 5 & 8 & 10 & 10 \\
\hline min_samples_leaf & 3 & 5 & 4 & 5 & 10 & 2 \\
\hline learning_rate & 0.05 & 0.05 & 0.06 & 0.05 & 0.05 & 0.05 \\
\hline min_samples_split & 2 & 4 & 3 & 2 & 4 & 5 \\
\hline Ioss & Is & Is & Is & Is & Is & huber \\
\hline subsample & 0.3 & 0.3 & 0.8 & 0.3 & 0.5 & 0.5 \\
\hline
\end{tabular}


The number of proteins for each group was determined according to the error score of the $\mathrm{K}$ means clustering algorithm. This number indicates the number of proteins obtained when the error rate is the smallest. Fig 3. and Fig. 4 show comparisons of cluster result between the first 30 selected proteins and the selected proteins when the error rate is the smallest. In Fig. 3 and Fig. 4, the comparison cluster result for each group is shown side by side. When cluster results are examined, it is seen that the proteins affected by memantine or RO4938581 for each group cluster better in the number of proteins selected. These figures reveal how important the selected proteins are.
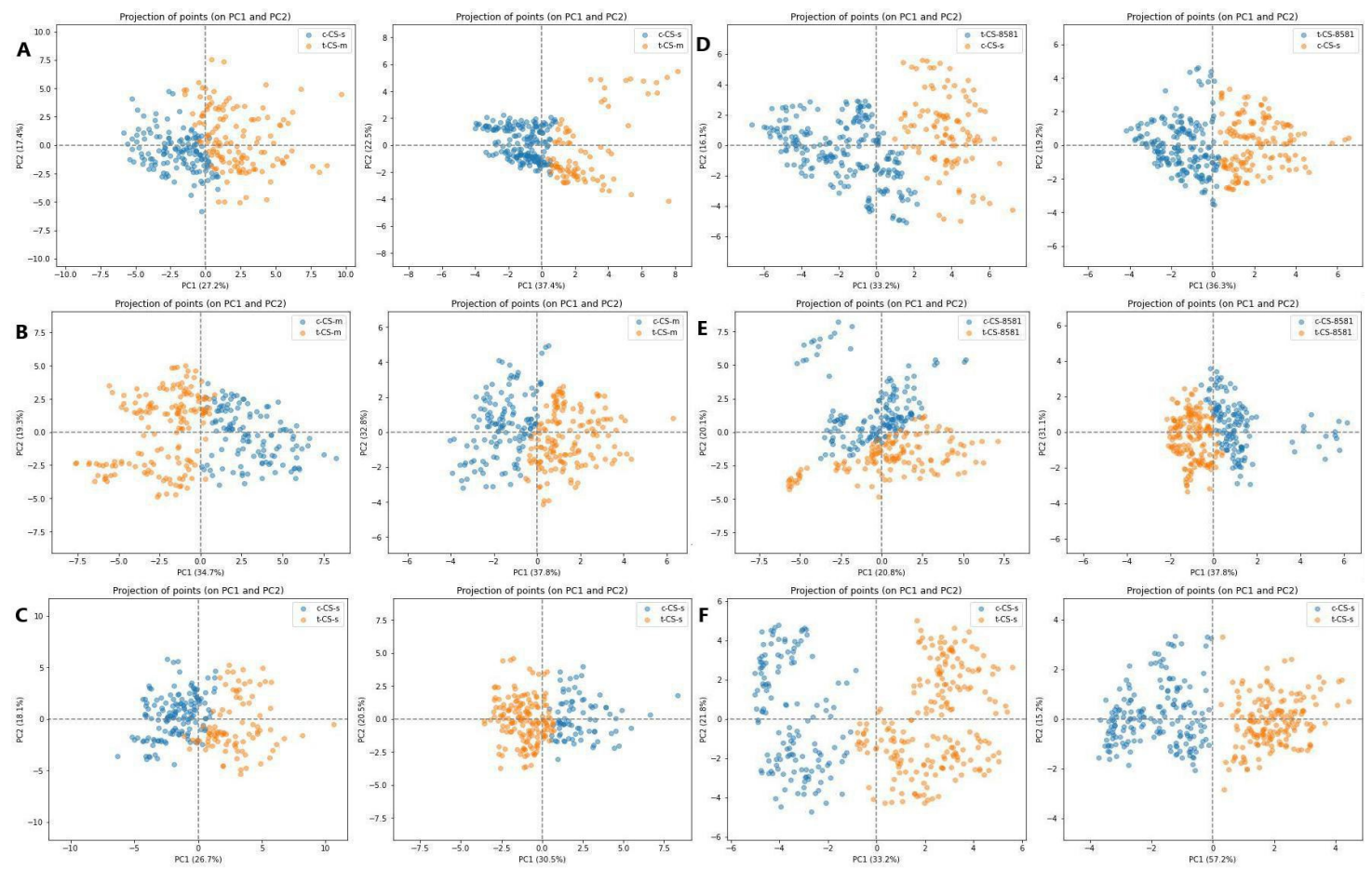

Fig 3. Comparisons of cluster result between the first $\mathbf{3 0}$ selected proteins and the selected proteins. a,b,c, The comparison result for Groups 1,2,3 in the memantine dataset, respectively, d,e,f, The comparison result for Groups 1,2,3 in the RO4938581 dataset, respectively.
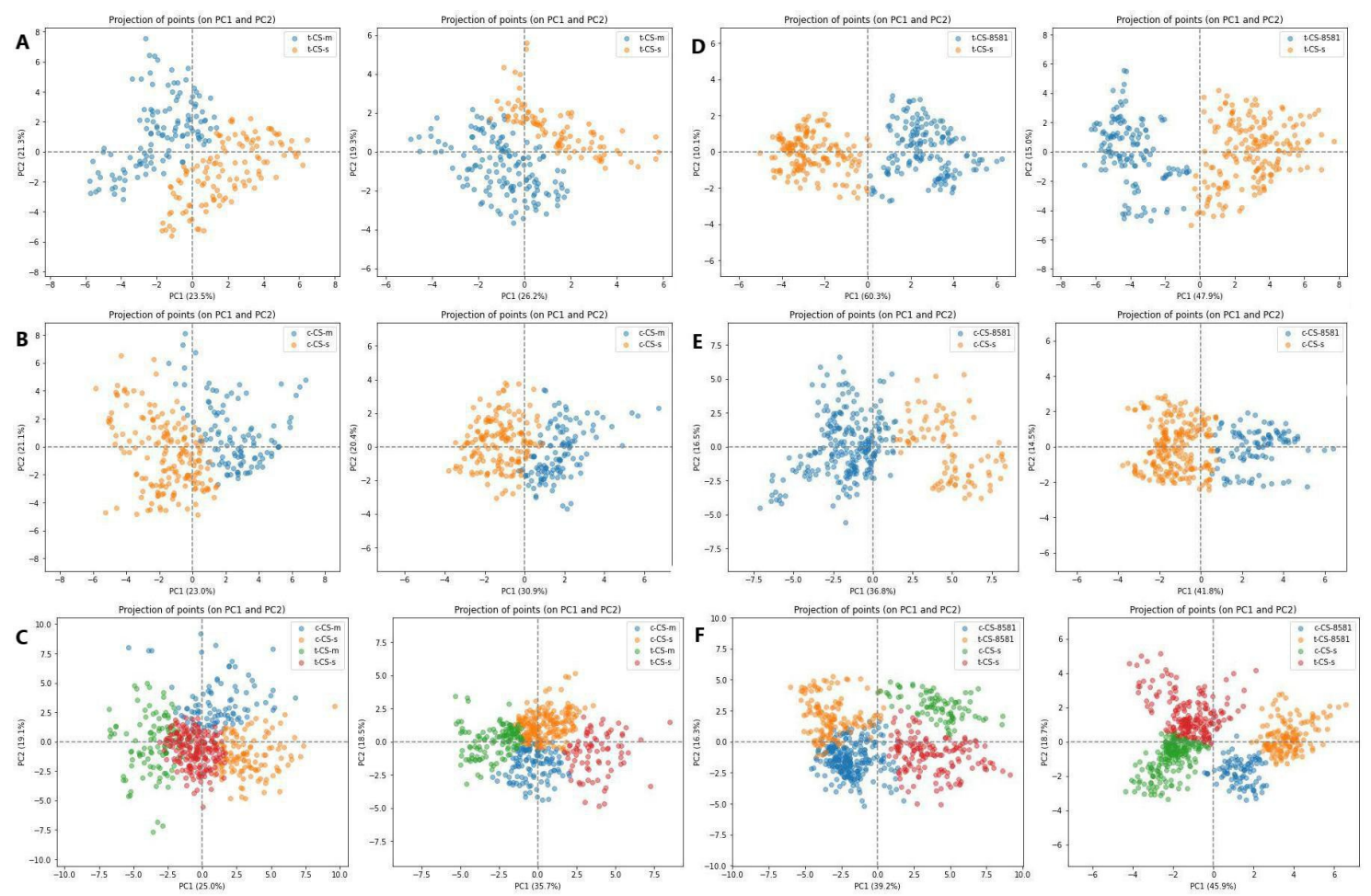

Fig 4. Comparisons of cluster result between the first 30 selected proteins and the selected proteins. a,b,c, The comparison result for Groups 4,5,6 in the memantine dataset, respectively, d,e,f, The comparison result for Groups 4,5,6 in the RO4938581 dataset, respectively. 
Table 3 and Table 4 show the proteins selected for each group in the memantine and RO4938581 datasets, respectively. When the tables are compared, it appears that different proteins are selected for the two datasets in which different drugs act. This condition can be caused by the protein content in each dataset or the interaction of drugs with different receptors of cell surface.

Table 3. A subset of proteins selected for each group obtained from the memantine dataset

\begin{tabular}{|c|c|c|c|c|c|c|}
\hline Selected proteins & Group 1 & Group 2 & Group 3 & Group 4 & Group 5 & Group 6 \\
\hline 1 & AMPKA & MTOR & Tau & S6 & pPKCAB & APP \\
\hline 2 & APP & pMTOR & S6 & GluR3 & Tau & P38 \\
\hline 3 & BRAF & APP & APP & BRAF & nNOS & MTOR \\
\hline 4 & Tau & P38 & RAPTOR & nNOS & pJNK & GluR3 \\
\hline 5 & pNUMB & CaNA & AMPKA & NUMB & pAKT & Tau \\
\hline 6 & DYRK1A & BRAF & GluR3 & pP70S6 & IL1B & NR2B \\
\hline 7 & ERK & pPKCAB & DYRK1A & pCAMKII & BAX & H3AcK18 \\
\hline 8 & BAX & NR2B & PKCA & Tau & Ubiquitin & PKCA \\
\hline 9 & pERK & DYRK1A & P38 & P38 & CaNA & BRAF \\
\hline 10 & ADARB1 & & Ubiquitin & SNCA & AcetylH3K9 & NR2A \\
\hline 11 & IL1B & & pNUMB & AcetylH3K9 & SHH & CaNA \\
\hline 12 & nNOS & & AcetylH3K9 & Ubiquitin & & AMPKA \\
\hline 13 & pNR1 & & Bcatenin & PSD95 & & RAPTOR \\
\hline 14 & & & & CDK5 & & pPKCAB \\
\hline 15 & & & & & & DSCR1 \\
\hline
\end{tabular}

Table 4. A subset of proteins selected for each group obtained from the RO4938581 dataset

\begin{tabular}{|c|c|c|c|c|c|c|}
\hline Selected proteins & Group 1 & Group 2 & Group 3 & Group 4 & Group 5 & Group 6 \\
\hline 1 & ERBB4 & H3AcLys27 & APP & ADARB1 & ERBB4 & APP \\
\hline 2 & ADARB1 & DONSON & BAD & BAX & ADARB1 & SHH \\
\hline 3 & SOD1 & RCAN1 & RCAN1 & AMPKA & pELK & BAD \\
\hline 4 & IL1B & pELK & GluR4 & TRKA & S6 & RCAN1 \\
\hline 5 & DONSON & APP & pS6 & BRAF & ARC & RSK \\
\hline 6 & STAT3 & ERBB4 & pEIFB4 & STAT3 & pS6 & GluR3 \\
\hline 7 & pELK & & BCL2 & CASP3 & pNR2B & DONSON \\
\hline 8 & RCAN1 & & IL1B & CFOS & BRAF & CASP3 \\
\hline 9 & BDNF & & & JNK & CASP3 & ITSN1 \\
\hline 10 & H3ACLys27 & & & H3AcLys27 & PCREB & pS6 \\
\hline 11 & P70S6 & & & DONSON & pNR2A & pELK \\
\hline 12 & pRSK & & & pS6 & & GFAP \\
\hline 13 & & & & ERK & & CDK5 \\
\hline 14 & & & & TAU & & \\
\hline
\end{tabular}

\section{Scenario result.}

After the protein subsets were determined, the effects of memantine and RO4938581 were interpreted using GSEA to reveal the biological processes acting on DS due to changes in expression levels. The common GO terms were found in three groups: Group 1, Group 2 and Group 3 and are shown in Table 5. The GO terms indicate the relationships between a gene product to biological process (BP), molecular function (MF) and cellular component (CC). The first and second column of Table 5 show GO term ID and the types of GO terms which can be BP, MF, CC, respectively. The enrichment column indicates whether there are GO terms ( $p$-value $<0.05$ ) that indicate significantly enriched biological processes. The last column indicates the name of the biological process. Group 1 and Group 4, respectively, show a comparison of the levels of protein expression of the rescued learning process with the normal learning process and with the unsuccessful learning process. Group 6 highlights the difference in protein expression between the trisomy and control groups. BP terms have been evaluated to identify impaired biological processes in DS due to changes in the expression levels of selected proteins. To better understand the mechanisms of drugs and provide clues for drug interaction and the future prediction of drug target interaction, drug targets are associated with biological functions by analyzing the MF terms. 
Table 5. Common GO terms in which both drugs act

\begin{tabular}{|c|c|c|c|c|}
\hline \multirow{8}{*}{ Group 1} & GO & NS & Enrichment & Name \\
\hline & GO:0018105 & $\mathrm{BP}$ & $\mathrm{e}$ & peptidyl-serine phosphorylation \\
\hline & GO:0045944 & $\mathrm{BP}$ & e & positive regulation of transcription by RNA polymerase II \\
\hline & GO:0004674 & MF & $\mathrm{e}$ & protein serine/threonine kinase activity \\
\hline & GO:0106311 & MF & $\mathrm{e}$ & protein threonine kinase activity \\
\hline & GO:0106310 & MF & $\mathrm{e}$ & protein serine kinase activity \\
\hline & GO:0004672 & MF & $\mathrm{e}$ & protein kinase activity \\
\hline & GO:0005524 & MF & $\mathrm{e}$ & ATP binding \\
\hline \multirow{10}{*}{ Group 4} & GO:0018105 & $\mathrm{BP}$ & e & peptidyl-serine phosphorylation \\
\hline & GO:0051403 & $\mathrm{BP}$ & $\mathrm{e}$ & stress-activated MAPK cascade \\
\hline & GO:0006468 & $\mathrm{BP}$ & e & protein phosphorylation \\
\hline & GO:0007049 & $\mathrm{BP}$ & e & cell cycle \\
\hline & GO:0048511 & $\mathrm{BP}$ & $\mathrm{e}$ & rhythmic process \\
\hline & GO:0106311 & MF & $\mathrm{e}$ & protein threonine kinase activity \\
\hline & GO:0106310 & MF & $\mathrm{e}$ & protein serine kinase activity \\
\hline & GO:0004707 & MF & $\mathrm{e}$ & MAP kinase activity \\
\hline & GO:0005524 & MF & $\mathrm{e}$ & ATP binding \\
\hline & GO:0004708 & MF & $\mathrm{e}$ & MAP kinase kinase activity \\
\hline \multirow{3}{*}{ Group 6} & GO:0018105 & $\mathrm{BP}$ & e & peptidyl-serine phosphorylation \\
\hline & GO:0004674 & MF & $\mathrm{e}$ & protein serine/threonine kinase activity \\
\hline & GO:0106310 & MF & $\mathrm{e}$ & protein serine kinase activity \\
\hline
\end{tabular}

When the common GO terms of proteins selected from two different datasets in which two different drugs interact were examined, it was found that the peptide-serine phosphorylation process is very important for learning performance in DS. Thus, the function of two different drug targets is mainly related to the signal transduction. Also, protein serine kinase activity function can be effective in drug target interaction for DS. In addition, the result of Group 1 shows that the process of positive regulation of transcription by the RNA polymerase II process is effective in improving learning performance with drugs. The result of Group 4 shows that stress level, rhythmic process, protein phosphorylation and cell cycle are effective biological processes for learning.

\section{Discussion}

Associating genes with the diseases they are associated with is one of the most difficult situations encountered in the medical field. Disruptions in cellular processes lead to the onset of the disease and are considered to occur decades before the first symptoms of the disease. By precisely determining cellular processes using high-level GO terms, the relationship of genes to diseases can be found, and also the bridge for associating the necessary drug with proteins can be determined. In this paper, an algorithmic approach has been developed to systematically investigate the applicability of GO terms in the interpretation of DSrelated disease datasets to improve the understanding how multiple genes lead to DS symptoms. Our algorithmic approach has led to several important findings. The most attractive is that, taking into account the protein-coding genes, Ts65Dn mice treated with different drugs show biological processes marked with the same GO terms that can be effective in learning.

DS arises because Hsa21 is a triplicate due to biological errors or because there is a Robertsonian translocation between chromosome 21 and another chromosome. Due to its high importance, scientists are trying to develop drugs for the relief of different symptoms of DS. However, the development of new drugs is a complex and expensive process due to the lack of effectiveness, which is associated with poor or biased target selection. In this study, the critical roles of proteins expressed by different drugs and which are potential targets of drugs were analyzed by comparing the protein expression levels of control mice and Ts65Dn mice. Ts65Dn mice cannot learn, but if drugs are injected into these mice, they can learn successfully and thus learning is rescued. By evaluating the protein expression level of Ts65Dn mice and control mice with or without drug injection, drug effects on DS can be investigated. Using the gradient boosting algorithm, significant protein subsets were selected from RO4938581 or memantine drug datasets. When selecting protein subsets, the highest accuracy score of PCA cluster analysis was taken into account. Subsequently, the common gene terms in which both drugs act on proteins were studied by performing GSEA. Protein subsets from the interaction of two different drugs with mice were evaluated together to understand the overall drug trend and to find out the biological processes by which the selected proteins in these two subsets are affected. In previous studies, the drug effects of memantine or RO4938581 were evaluated using statistical analysis methods and machine learning techniques, and important proteins that 
interact with the drug were identified. However, in these studies, the biological processes affected by drugs could not be determined by making the correct evaluation from protein to gene. Thus, in this rearch, in addition to selecting important protein subsets for two drug dataset, GSEA was done in order to indicate the association between drug compounds and GO terms. With enrichment analysis, a few candidate genes showing specific pattern of expression were selected for therapeutic approaches and thus effective GO terms were determined for therapeutic targets. The enrichment analysis of the two different drug targets showed that common GO-BP: peptidyl-serine phosphorylation and common GO-MF: protein serine kinase activity.

Despite the advancement in computational methods in drug target prediction, the mostly used methods such as docking are primarily based on the structural similarities of drugs rather than biological relevance. This study helps to delineate the molecular basis of DS to aid the development of diagnostic tools and treatments. Investigation of the association between $\mathrm{BP} / \mathrm{MF} \mathrm{GO}$ terms and drugs is helpful for discovering targets of drug compounds, thereby obtaining new drug effects. It is estimated that the GO terms, the importance of which is emphasized in this study, will play an effective role in the treatment of the learning process in DS.

\section{Methods}

\section{Data preprocessing.}

Both memantine and RO4938581 drug datasets have one or more mice with missing values. This can result to misleading predictions for the unknowns. Thus, the missing values are replaced with the average expression levels of the corresponding sample of that protein in the same class. For example, if one mouse is missing the first sample expression level information, it is replaced with the average value of first sample protein expression of other mice in the same class. With this replacement step, the effect of dilution ratio is considered. Also, because of $\% 50$ missing expression level information, the expresion level of seven proteins of RO4938581 drug dataset was not taken into account and discarded from the dataset. After replacement missing values with appropriate value explained above, all measurements are normalized with Z-score normalization to prevent proteins with higher values influence on the classification result erroneously. When normalizing data in different groups, attention was paid to the normalization of data containing only those classes of mice that should be present in the specified group. With Z-score normalization ${ }^{28}$ as stated below, mean of the scores is subtracted from each score and then divided into the standard deviation.

$$
\mathbf{Z}=\frac{x-\mu}{\sigma}
$$

\section{Selecting proteins with gradient boosting tree algorithm.}

In boosting algorithm, a strong learner is created by adding a new weak learner to each iteration. With each iteration, new models are built to overcome errors made in previous iterations. With the gradient boosting tree method, the first leaf is created first. After that, new trees are created taking into account prediction errors. This situation continues until the number of decided trees or more improvements cannot be made from the mode ${ }^{29-31}$. In the gradient tree boosting, gradient boosting is typically used with decision trees of a fixed size as base learners. Generic gradient boosting at the $\mathrm{m}$-th step would fit a decision tree $\mathrm{h}_{\mathrm{m}}(\mathrm{x})$ to the residuals as seen in below. The tree partitions the input space into disjoint regions according to number of its leaves and predicts a constant value in each region.

$$
h_{m}(x)=\sum_{j=1}^{J_{m}} b_{j m} \mathbf{1}_{R_{j+n}}(x)
$$

( $\mathrm{Jm}$ is number of leaves, $\mathrm{R}_{1 \mathrm{~m}}, \ldots . ., \mathrm{R}_{\mathrm{jm}}$ are disjoint regions, $\mathrm{b}_{\mathrm{jm}}$ is value predicted in the region $\mathrm{R}_{\mathrm{jm}}$ )

In the above equation, the coefficients $b_{\mathrm{jm}}$ are multiplied by some value $\gamma_{\mathrm{m}}$ to minimize the loss function.

$$
F_{m}(x)=F_{m-1}(x)+\sum_{j=1}^{J_{m}} \gamma_{j m} \mathbf{1}_{R_{j m}}(x), \quad \gamma_{j m}=\underset{\gamma}{\arg \min } \sum_{x_{i} \in R_{j m}} L\left(y_{i}, F_{m-1}\left(x_{i}\right)+\gamma\right)
$$

( Model F predicts the values and minimize loss function $\mathrm{L}(\mathrm{y}, \mathrm{F}(\mathrm{x})$ )) 
In the gradient tree boosting algorithm, the grid search technique creates a model for each possible combination of all hyperparameter values. The hyperparameters of gradient tree increment modeling are the maximum depth of each tree, the number of trees, the learning rate, and the minimum number of observations at the terminal nodes of the trees. The decisiveness of the grid search method ${ }^{32}$ is found by comparing the results according to different hyperparameter values. Each model in a different combination of parameters was evaluated and the model that gives the most accurate result was selected.

In grid search and gradient boosting steps, 5 fold cross validation was applied to evaluate the performance of the model. In 5 fold cross validation, part of the data that is not used to train the model is then used to test this sample. Thus, every observation in the dataset has the opportunity to appear in the training and test set.

\section{Selecting protein subset with PCA cluster analysis.}

After the protein subset is selected for each model, the best model is found by PCA cluster analysis. PCA is a method for finding the projection of data onto a low-dimensional axis in such a way as to maximize variance $^{33-37}$. With the Kmeans clustering algorithm, the data points are divided into k sets, where each data point belongs to the set with the nearest average, and the data can be effectively clustered with decreasing axis size.

The influence of all variables on the evaluation of biological processes can be deciphered. For example, when analyzing more than a hundred genes, it is necessary to add axis for each gene, which makes it mandatory to use more than a hundred axes. Thanks to the PCA method, data can be easily analyzed in two or three dimensions by including all genes.
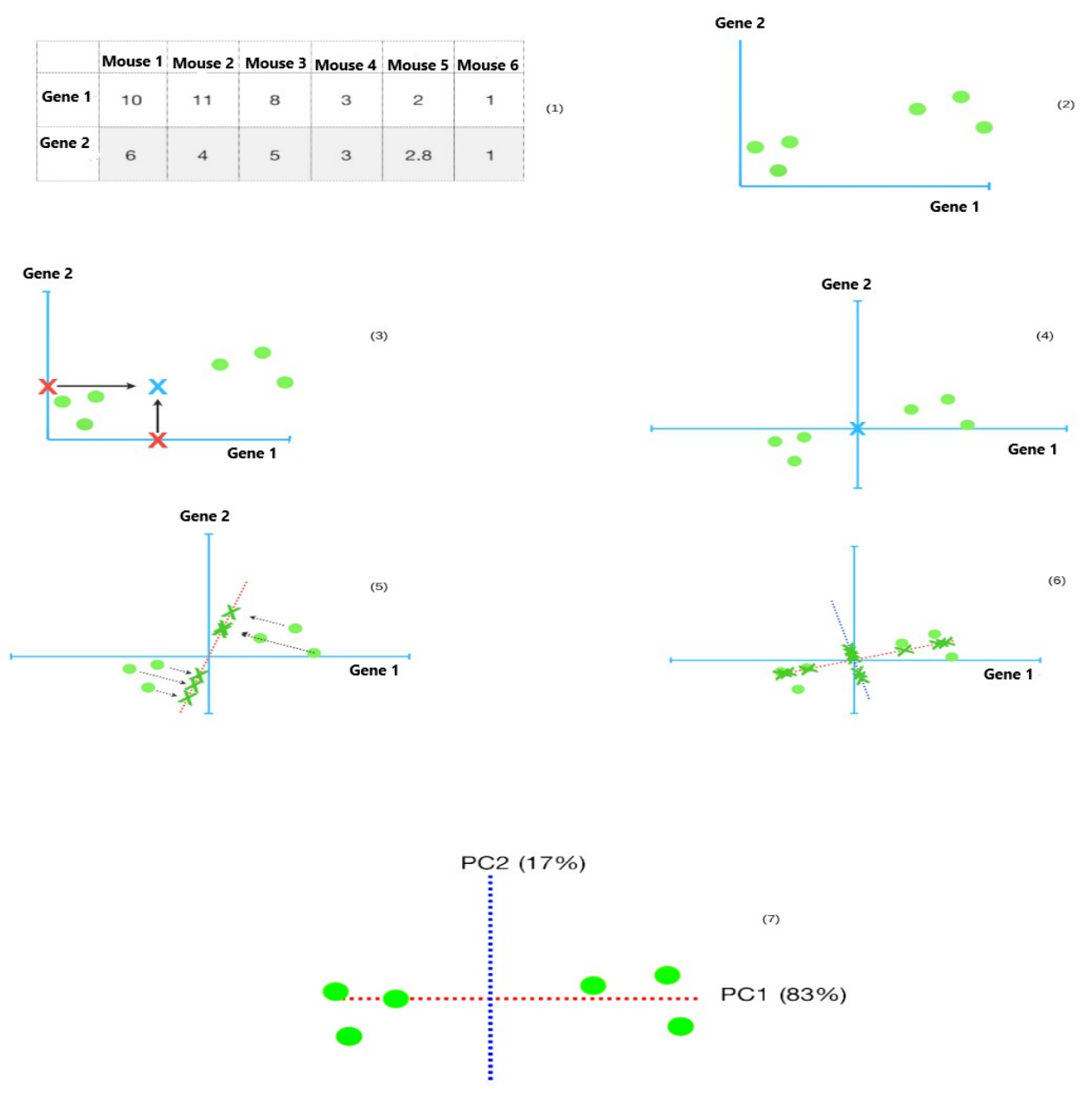

Fig 5. | PCA cluster analysis stages on a small dataset 
Fig 5. shows PCA cluster stages on a small dataset sample that includes 2 gene expression levels of 6 mice.

(1) shows the values of 2 gene measurements taken from 6 different mice in a table.

(2) shows the distribution of the protein expression of 6 different mice on a two-dimensional graph. The mouse numbers $1,2,3$ are clustered on the right side of the graph and the mouse numbers 4, 5, 6 are clustered on the left side of the graph. Mice with a similar amount of protein expression grouped together.

(3) The center point of the graph is calculated by taking the averages of the two axes.

(4) The data is shifted on the graph and the center point of the graph is shifted to the origin.

(5) A line has been drawn that will be at the point of leaving a trace of all the data and will pass at the closest distance to the center of the data. The goal here is to find the most accurate line so that this line is at the closest distance to the center of each data, and the projection distance of the data on the line to the origin is maximum. Thanks to this process, the variance is maximized.

(6) The second line (the blue line) perpendicular to the line calculated in the previous step (red line) is drawn. The red and blue lines become first principal component (PC1) and second component (PC2), respectively.

(7) shows the distribution of the data on the calculated PC1 and PC2 axes.

\section{Gene ontology.}

It is known that disorders of the cellular process emphasize the onset of the disease before clinical manifestations. Therefore, the BP terms are very important and indicate the onset of the disease. Also, in order to understand the mechanisms of drug target interaction effectively, MF terms must be evaluated to associate drug targets with biological functions. In previous works, AD, Parkinson's disease and autism were evaluated by conducting GSEA ${ }^{38-40}$. In this study, GSEA was performed and the drug tendency was evaluated by analyzing the common gene terms that both drugs affect on the selected proteins.

The datasets can be examined by GSEA analysis to reveal impaired biological processes in the disease due to changes in expression levels or regulation of these genes. The $p$-value is used to examine the significance of GO term enrichment with a modified Fisher's exact test. The Benjamini value is used to correct the enrichment $p$-values of individual term. The GO terms with a $\mathrm{p}$-value of $\leq 0.05$ and Benjamini value of $\leq 0.5$ are regarded as significant. This analysis helps to develop improved treatments for the treatment of the disease, as well as early diagnosis. GO analysis describes functions of protein. A typical protein is assigned three types of GO terms: BP describes the processes involving this protein, MF denotes the types of reactions induced by the protein and CC determines the location of the protein. Such annotation syntax helps to compare the functions of two proteins by measuring the terms similarity ${ }^{41-45}$.

\section{Data availability}

The memantine drug dataset is deposited to the Machine Learning Repository at University of California Irvine (Mice Protein Expression Dataset, https://archive.ics.uci.edu/ml/datasets/Mice+Protein+Expression\#) RO4938581 drug dataset was taken from Prof. Katheleen Gardiner.

\section{References}

1. Lejeune, J., Gautier, M. \& Turpin, R. Study of somatic chromosomes from 9 mongoloid children. $C R$ Hebd Seances Acad Sci 248(11), 1721-2 (1959).

2. Sturgeon, X. \& Gardiner, K. J. Transcript catalogs of human chromosome 21 and orthologous chimpanzee and mouse regions. Mammalian Genome 22, 261-271.3 (2011).

3. Antonarakis, S., Lyle, R., Dermitzakis, E., Reymond, A. \& Deutsch, S. Chromosome 21 and Down syndrome: From genomics to pathophysiology. Nature reviews Genetics 5, 725-38 (2004).

4. Elton, T. S., Sansom, S. E. \& Martin, M. M. Trisomy-21 gene dosage overexpression of miRNAs results in the haploinsufficiency of specific target proteins. RNA Biol 7, 540-547 (2010).

5. Lu, J. \& Sheen, V. Genetic and Epigenetic Mechanisms in Down Syndrome Brain: Down Syndrome Ch. 12 ( InTech Editor. Subrata Dey, 2013).

6. Chapman, R.\& Hesketh, L. Language, cognition, and short-term memory in individuals with Down syndrome. Down's syndrome, research and practice 7, 1-7 (2001). 
7. Pennington, B., Moon, J., Edgin, J., Stedron, J. \& Nadel, L. The Neuropsychology of Down Syndrome: Evidence for Hippocampal Dysfunction. Child development 74, 75-93 (2003).

8. Dierssen, M., Herault, Y. \& Estivill, X. Aneuploidy: From a Physiological Mechanism of Variance to Down Syndrome. Physiological reviews 89, 887-920 (2009).

9. Contestabile, A., Benfenati, F. \& Gasparini, L. Communication breaks-Down: From neurodevelopment defects to cognitive disabilities in Down syndrome. Progress in neurobiology 91, 1-22 (2010).

10. Gardiner, K., Herault, Y., Lott, I., Antonarakis, S., Reeves, R. \& Dierssen, M. Down Syndrome: From Understanding the Neurobiology to Therapy. The Journal of Neuroscience 30, 14943-5 (2010).

11. Davisson, M. T. Segmental trisomy as a mouse model for Down syndrome. Prog Clin Biol Res 384, 117133 (1993).

12. Rueda, N., Flórez, J. \& Martínez-Cué, C. Mouse Models of Down Syndrome as a Tool to Unravel the Causes of Mental Disabilities. Neural plasticity 2012, 584071. (2012).

13. Gardiner, K.J. Pharmacological approaches to improving cognitive function in Down syndrome: current status and considerations. Drug Design, Development and Therapy 9, 103-125 (2015).

14. Kamat, P.K. et al. Okadaic acidinduced Tau phosphorylation in rat brain: role of NMDA receptor. Neuroscience 238, 97-113 (2013).

15. Costa, A.C., Scott-McKean, J.J. \& Stasko, MR. Acute injections of the NMDA receptor antagonist memantine rescue performance deficits of the Ts65Dn mouse model of Down syndrome on a fear conditioning test. Neuropsychopharmacology 33, 1624-1632 (2008).

16. Chang, Q., \& Gold, P. E. Age-related changes in memory and in acetylcholine functions in the hippocampus in the Ts65Dn mouse, a model of Down syndrome. Neurobiol Learn Mem 89, 167-17 (2008).

17. Gardiner, K. J. Molecular basis of pharmacotherapies for cognition in Down syndrome. Trends in pharmacological sciences 31, 66-73 (2010).

18. Chen, H.S. \& Lipton, S.A. Pharmacological implications of two distinct mechanisms of interaction of memantine with N-methyl-D-aspartate-gated channels. J Pharmacol Ex. Ther 314, 961-97 (2005).

19. Lipton, S.A. Pathologically-activated therapeutics for neuroprotection: mechanism of NMDA receptor block block by memantine and S-nitrosylation. Curr Drug Targets 8, 621-632 (2007).

20. David, O. et al. N-Methyl D-Aspartate (NMDA) Receptor Antagonists and Memantine Treatment for Alzheimer's Disease, Vascular Dementia and Parkinson's Disease. Curr Alzheimer Res 9, 746-758 (2012).

21. Larranaga, P. et al. Machine learning in bioinformatics. Brief Bioinform 7(1),86-112 (2006).

22. Braudeau, J., Delatour, B. \& Duchon, A. Specific targeting of the GABAA receptor 5 subtype by a selective inverse agonist restores cognitive deficits in Down syndrome mice. Journal of Psychopharmacology 25(8), 1030-1042 (2011).

23. Block, A. et al. The GABAA 5-selective Modulator, RO4938581, Rescues Protein Anomalies in the Ts65Dn Mouse Model of Down Syndrome. Neuroscience 372, 192-212 (2017).

24. Ahmed, M.M. et al. Protein dynamics associated with failed and rescued learning in the Ts65Dn mouse model of Down syndrome. PLoS ONE 10(3), e0119491 (2015).

25. Higuera, C., Gardiner, K. J. \& Cios, K.J. Organizing Feature Maps Identify Proteins Critical to Learning in a Mouse Model of Down Syndrome. PLoS ONE 10(6), e0129126 (2015).

26.Fanselow, M.S. Factors governing one-trial contextual conditioning. Anim Learn Behav. 18(3), 264-70 (1990).

27.Tibes, R. et al. Reverse phase protein array: validation of a novel proteomic technology and utility for analysis of primary leukemia specimens and hematopoietic stem cells. Mol. Cancer Ther. 5, 2512-21 (2006). 28.Abdi, H. \& Williams, L.J. Normalizing data. In: Neil Salkind, editor. Encyclopedia of research design 935-8 (2010).

29. Fafalios, S., Charonyktakis, P., \& Tsamardinos, I. Gradient Boosting Trees. (2020).

30. Llew, M., Jonathan, B., Peter, B., \& Marcus, F. Boosting algorithms as gradient descent. MIT Press $512-$ 518 (1999).

31. Natekin, A., Knoll, A. Gradient boosting machines. Front Neurorobot 7, 21(2013).

32. Chicco, D. Ten quick tips for machine learning in computational biology. BioData Mining 10(35),35 (2017).

33. Alpaydin, E. Introduction to machine learning. MIT Press 113-120 (2010).

34. Miranda, A. A., Borgne, Y. A. \& Bontempi, G. New Routes from Minimal Approximation Error to Principal Components. Neural Processing Letters, Springer 27, 3 (2008).

35. Hotelling, H. Analysis of a complex of statistical variables into principal components. Journal of Educational Psychology 24, 498-520 (1933).

36. Hotelling, H. Relations between two sets of variates. Biometrika 28, 321-377 (1936) 
37.Leznik, M. \& Tofallis, C. Estimating Invariant Principal Components Using Diagonal Regression Preprint at https://www.semanticscholar.org/paper/Estimating-Invariant-Principal-Components-Using-Leznik-Tofallis /f6d6d6c50e9381f1a6c40f2b2167ed3ac652ef27 (2005).

38. Kramarz, B. et al. Improving the Gene Ontology Resource to Facilitate More Informative Analysis and Interpretation of Alzheimer's Disease Data. Genes 9(12), 593 (2018).

39. Foulger, R.E. et al. Using the Gene Ontology to annotate key players in Parkinson's disease. Neuroinformatics 14, 297-304 (2016) .

40. Patel, S., Roncaglia, P. \& Lovering, R.C. Using Gene Ontology to describe the role of the neurexinneuroligin-SHANK complex in human, mouse and rat and its relevance to autism. BMC Bioinformatics 16, 186 (2015).

41. Dat, D. et al. Evaluating Representations for Gene Ontology Terms. bioRxiv Preprint at https:// doi .org / 10.1101/765644 (2019).

42. Gene Ontology Consortium Expansion of the gene ontology knowledgebase and resources. Nucleic acids research 45, D331-D338 (2017).

43. Mazandu, G.K. \& Mulder, N.J. Information content-based gene ontology semantic similarity approaches: toward a unified framework theory. BioMed Res Int 2013, 292063 (2013).

44. Resnik, P. Semantic similarity in a taxonomy: An information-based measure and its application to problems of ambiguity in natural language. J. Artif. Intell. Res. 11, 95-130 (1999).

45. Yang, H., Nepusz, T. \& Paccanaro, A. Improving go semantic similarity measures by exploring the ontology beneath the terms and modelling uncertainty. Bioinformatics 28(10), 1383-1389 (2012).

\section{Acknowledgement}

I thank Prof. Katheleen Gardiner to provide RO4938581 drug dataset. 